\title{
Roflumilast, a type of phosphodiesterase 4 inhibitor, can reduce intestinal injury caused by sepsis
}

\author{
ZHONGYUAN ZHANG $^{1}$, MEIFENG LIANG ${ }^{2}$ and XIONGFEI WAN ${ }^{3}$ \\ ${ }^{1}$ Department of Pharmacy, Tianyou Hospital Affiliated to Wuhan University of Science and \\ Technology, Wuhan, Hubei 430060; Departments of ${ }^{2}$ Medical Insurance and Management and \\ ${ }^{3}$ Pharmacy, CR \& WISCO General Hospital, Wuhan, Hubei 430000, P.R. China
}

Received April 21, 2021; Accepted August 17, 2021

DOI: $10.3892 /$ etm.2021.10834

\begin{abstract}
Sepsis is a systemic inflammatory response syndrome caused by infection, which has a complex mechanism. The gastrointestinal tract is commonly the first organ affected by sepsis, but intestinal disease itself can also induce sepsis. Roflumilast has been found to exert anti-inflammatory effects and, thus, the present study sought to examine its effect on intestinal damage caused by sepsis. In vivo studies were conducted using cecal ligation and puncture rat models, and in vitro experiments were performed using IEC- 6 cells. The intestinal cells were first induced with lipopolysaccharide and the induced cells were then treated with roflumilast to evaluate its effects on phosphodiesterase (PDE)4 expression, intestinal function indices, release of inflammatory factors and cell apoptosis. The expression level of PDE4 in the small intestinal tissue of septic rats was found to be significantly higher compared with that in the normal group, suggesting that PDE4 may play a key role in intestinal injury caused by sepsis. It was found that roflumilast reduced PDE4 expression, as well as the levels of intestinal function indices, including lactate dehydrogenase, diamino oxidase and intestinal fatty acid-binding protein, in intestinal cells. Moreover, roflumilast reduced cellular damage, the release of inflammatory factors and apoptosis. In summary, the findings of the present study indicated that roflumilast can relieve the inflammation and apoptosis of intestinal cells caused by sepsis and can promote their functional recovery. These findings may promote the expansion of the clinical application of roflumilast in the future.
\end{abstract}

Correspondence to: Professor Zhongyuan Zhang, Department of Pharmacy, Tianyou Hospital Affiliated to Wuhan University of Science and Technology, 9 Tujialing, Dingziqiao Road, Wuchang, Wuhan, Hubei 430060, P.R. China

E-mail: zhangzyzhongyuan@163.com

Key words: roflumilast, phosphodiesterase 4, intestinal injury, sepsis, IEC-6 cells

\section{Introduction}

Sepsis is a systemic inflammatory response syndrome caused by infection. Essentially, sepsis is the body's response to infectious factors (1). The incidence of sepsis is high, with $>30$ million cases of severe sepsis annually worldwide and a continually increasing number every year (2). Despite the significant progress achieved in anti-infection treatment and organ function support technology in recent years (3), the fatality rate of sepsis remains high at $\sim 35 \%$. The high cost of sepsis treatment and the high demand for medical resources severely affect the quality of life of the patients, and sepsis poses a significant threat to human health. Sepsis can be caused by infection in any part of the human body, particularly in the gastrointestinal tract. The gastrointestinal tract is the first organ affected by sepsis, resulting in functional damage (4). Moreover, intestinal disease itself can also induce sepsis and lead to multiple organ dysfunction (5). Therefore, it is crucial to study the association between sepsis and the intestine, and to develop methods for alleviating the damage in intestinal function.

Phosphodiesterases (PDEs) can hydrolyze intracellular cyclic adenosine monophosphate (cAMP) or cyclic guanosine monophosphate (cGMP) (6), thereby blocking the biochemical effects mediated by these second messengers (7). cAMP and cGMP serve important roles in regulating cell activities. PDEs are widely distributed in the human body, and their physiological effects have been determined in numerous research fields (8). In recent years, PDEs have been attracting increased attention as new therapeutic targets and have become a new research hotspot. The clinical research of selective PDE4 and PDE5 inhibitors has also received significant attention $(9,10)$. PDE4 has multiple isozymes, which are divided into four subtypes: PDE4A, B, C and D (11). Furthermore, PDE4 has been found to be associated with cAMP hydrolysis in numerous types of inflammatory cells (12). Therefore, inhibiting PDE4 may help inhibit the physiological functions of inflammatory cells. More importantly, it was found that PDE4B knockdown could relieve intestinal dysregulation, bacterial overgrowth and endotoxemia (13).

Roflumilast is a highly selective PDE4 inhibitor used for the treatment of severe chronic obstructive pulmonary disease (COPD), and has been found to reduce the expression levels 
of pro-inflammatory factors in patients with COPD (14). Roflumilast has important anti-inflammatory properties, and the latest research has shown that roflumilast can reduce sepsis-induced lung (15), liver (14) and renal (16) tissue injury. However, whether roflumilast can reduce intestinal tissue damage induced by sepsis remains unknown.

Currently available research suggests that roflumilast can reduce the release of inflammatory factors in ulcerative colitis, decrease inducible nitric oxide synthase levels and enhance the expression of cAMP (17). Therefore, the aim of the present study was to investigate whether roflumilast could reduce sepsis-induced intestinal injury.

\section{Materials and methods}

Cell culture. IEC-6 cells (a rat small intestinal epithelial cell line) were purchased from the American Type Culture Collection. The cells were cultured in DMEM (Gibco; Thermo Fisher Scientific, Inc.) supplemented with 10\% FBS (Gibco; Thermo Fisher Scientific, Inc.), $10 \mathrm{U} / \mathrm{ml}$ insulin and $100 \mathrm{U} / \mathrm{ml}$ penicillin and streptomycin (Invitrogen; Thermo Fisher Scientific, Inc.), and maintained at $37^{\circ} \mathrm{C}$ in an incubator with $5 \% \mathrm{CO}_{2}$. The cells were treated with lipopolysaccharide (LPS; Sigma-Aldrich; Merck KGaA) for 24 h to construct the intestinal injury cellular model. Roflumilast (AdooQ Bioscience) was initially diluted to four concentrations, namely $1,5,10$ and $50 \mu \mathrm{g} / \mathrm{ml}$. In subsequent experiments, $5 \mu \mathrm{g} / \mathrm{ml}$ represented the low dose and $10 \mu \mathrm{g} / \mathrm{ml}$ the high dose.

Model establishment. A total of 15 male Sprague-Dawley rats (weight, 250-300 g; age, 8 weeks) were purchased from Jiangsu ALF Biotechnology Co., Ltd. and kept at $\sim 25^{\circ} \mathrm{C}$ under a 12-h light/dark cycle and $\sim 55 \%$ relative humidity, with free access to food and water. The rats were anesthetized using isoflurane inhalation (induction dose, 4\%; maintenance dose, $1.5 \%$ ) before cecal ligation and puncture (CLP), and their normal body temperature was maintained using heating pads. Briefly, the procedure was as follows: Laparotomy was performed, followed by ligation of the bowel below the ileocecal valve. The cecum was pierced twice using a needle, and then the abdominal cavity was closed and the rats were resuscitated. Prior to collecting the intestinal tissues, the rats were euthanized with intraperitoneal injection of $200 \mathrm{mg} / \mathrm{kg}$ pentobarbital sodium followed by cervical dislocation. All the experiments were conducted according to the institutional criteria for the care and use of laboratory research animals and all efforts were made to minimize animal suffering.

$H \& E$ staining. The small intestinal tissues of rats were rinsed, fixed in $4 \%$ paraformaldehyde at room temperature overnight, dehydrated and embedded in paraffin. The sections were then stained with hematoxylin solution for $10 \mathrm{~min}$ at room temperature followed by eosin solution for $1 \mathrm{~min}$ at room temperature. After rinsing with $70 \%$ alcohol, an ascending alcohol gradient was used for dehydration and xylene was used to transparentize the sections. The sections were then sealed using neutral gum. The physiological changes of the tissues were observed under a light microscope (magnification, x200; Olympus Corporation) and an appropriate field of view was selected to capture the image.
Intestinal function index detection. Small intestinal tissues were lysed using RIPA lysis buffer, and tissue samples were used to detect the activity of lactate dehydrogenase (LDH), D-amino acid oxidase (DAO) and intestinal fatty acid-binding protein (iFABP). The assays were conducted using an LDH Assay kit (cat. no. ab197004; Abcam), DAO Activity Assay kit (cat. no. ab273325; Abcam) and iFABP ELISA kit [cat. no. JK-(a)-5035; Jingkang Biotechnology Co. Ltd.], according to the manufacturer's instructions.

Reverse transcription-quantitative PCR (RT-qPCR) analysis. Total RNA was isolated from small intestinal tissue and IEC-6 cells using the RNAsimple total RNA kit (Tiangen Biotech Co., Ltd.), and cDNA was obtained using a Reverse Transcriptase kit (Invitrogen; Thermo Fisher Scientific, Inc.) according to the manufacturer's instructions. qPCR was performed using the QuantiTect SYBR-Green PCR kit (Qiagen, Inc.). The thermocycling condition was as follows: $95^{\circ} \mathrm{C}$ For $2 \mathrm{~min}$, followed by 40 cycles at $95^{\circ} \mathrm{C}$ for $10 \mathrm{sec}, 60^{\circ} \mathrm{C}$ for $35 \mathrm{sec}$ and $72^{\circ} \mathrm{C}$ for $10 \mathrm{sec}$. The $2^{-\triangle \Delta \mathrm{Cq}}$ method (18) was applied for data analysis, with normalization to GAPDH. The primer sequences are listed in Table I.

Western blotting. Total protein was extracted from small intestinal tissue and IEC- 6 cells and homogenized in RIPA lysis buffer (Beyotime Institute of Biotechnology). After protein quantification was conducted using a BCA protein assay kit (Glpbio Technology, Inc.), the same amount of $30 \mathrm{mg}$ protein was used for electrophoresis, and the protein separated by 10 or $12 \%$ SDS-polyacrylamide gel electrophoresis was transferred to PVDF membranes via electroporation. The membranes were first incubated in 5\% skimmed milk for $2 \mathrm{~h}$ at room temperature, and then incubated with primary antibodies against PDE4A (cat. no. ab14628; 1:1,000; Abcam), PDE4B (cat. no. ab170939; 1:1,000; Abcam), PDE4D (cat. no. ab114613; 1:1,000; Abcam), p65 (cat. no. ab32536; 1:1,000; Abcam), histone H3 (cat. no. ab1791; 1:1,000; Abcam), $\beta$-actin (cat. no. ab8226; 1:1,000; Abcam), Bax (cat. no. ab32503; 1:1,000; Abcam), cleaved caspase 3 (cat. no. ab32042; 1:1,000; Abcam), cleaved poly(ADP-ribose) polymerase 1 (PARP; cat. no. ab32064; 1:1,000; Abcam) and Bcl-2 (cat. no. ab32124; 1:1,000; Abcam) at $4^{\circ} \mathrm{C}$ overnight. Then, the membranes were washed with $0.05 \%$ TBS-Tween 20 , and were incubated with HRP-conjugated anti-rabbit antibody (cat. no. 65-6120; 1: 10,000; Thermo Fisher Scientific, Inc.). The strip was developed using an ECL reagent (Thermo Fisher Scientific, Inc.), and ImageJ software (v1.8; National Institutes of Health) was used to analyze the gray value.

When detecting the nuclear and cytoplasmic protein levels, the cells were centrifuged at $12,000 \mathrm{xg}$ for $5 \mathrm{~min}$ at $4^{\circ} \mathrm{C}$. The cytoplasmic protein extract was added to the pellet, which was then centrifuged at $12,000 \mathrm{xg}$ at $4^{\circ} \mathrm{C}$, and this supernatant was considered as the cytoplasmic protein lysis solution. The cells were centrifuged at $12,000 \times \mathrm{g}$ for $1 \mathrm{~min}$ at $4^{\circ} \mathrm{C}$, the nuclear lysate was added and sample was centrifuged at $12,000 \times \mathrm{xg}$ for $5 \mathrm{~min}$ at $4^{\circ} \mathrm{C}$, after being vortexed several times. This supernatant was considered as the nuclear protein lysate.

Cell Counting Kit-8 (CCK-8) assay. The cells (5x103/well) were added to a 96-well plate and cultured in an incubator with $5 \% \mathrm{CO}_{2}$ at $37^{\circ} \mathrm{C}$. The CCK-8 solution (Beyotime Institute 
Table I. Primer sequences used for reverse transcription-quantitative PCR analysis.

\begin{tabular}{ll}
\hline Gene & \multicolumn{1}{c}{ Sequence (5'-3') } \\
\hline PDE4A sense & TTCTGCAAGAGGGAGACAGGA \\
PDE4A antisense & GAGTGTCCTCCTCGCTGAAG \\
PDE4B sense & GGCCAGGCTTTGCTTACTGT \\
PDE4B antisense & ATTTGGGAAGCCGTGATGGT \\
PDE4D sense & TCAAAGCCCCCAAGCATCTCTG \\
PDE4D antisense & GTCTGAGTCCCTGGAAAGACG \\
TNF- $\alpha$ sense & TTCCCAAATGGGCTCCCTCT \\
TNF- $\alpha$ antisense & GTGGGCTACGGGCTTGTCAC \\
IL-1 $\beta$ sense & TGCCACCTTTTGACAGTGATG \\
IL-1 $\beta$ antisense & ATGTGCTGCTGCGAGATTTG \\
IL-6 sense & TCTGGGAAATCGTGGAAATGAG \\
IL-6 antisense & TCTCTGAAGGACTCTGGCTTTGTC \\
GAPDH sense & GCATCTTCTTGTGCAGTGCC \\
GAPDH antisense & GATGGTGATGGGTTTCCCGT
\end{tabular}

PDE, phosphodiesterase.

of Biotechnology) was added after cells were treated with different concentrations of LPS or additional roflumilast for $24 \mathrm{~h}$. The cells were cultured for $1 \mathrm{~h}$ and then the optical density was measured at $450 \mathrm{~nm}$ using a microplate reader (Thermo Fisher Scientific, Inc.).

TUNEL assay. Cells (5x10 $/$ well) were seeded into a $24-w e l l$ plate and cultured until they reached $\sim 80 \%$ confluence. The cells were fixed with $4 \%$ paraformaldehyde for $30 \mathrm{~min}$ at room temperature and permeated with PBS containing $0.3 \%$ Triton X-100 for another $5 \mathrm{~min}$ at room temperature. Following blocking with $3 \% \mathrm{H}_{2} \mathrm{O}_{2}$ for $5 \mathrm{~min}$ at room temperature, TUNEL staining was performed according to the procedure provided in the TUNEL assay kit (Beyotime Institute of Biotechnology). DAPI was used to counterstain the nuclei for $10 \mathrm{~min}$ at room temperature. The results were observed at five random fields of view under a fluorescence microscope (magnification, x200; Olympus Corporation).

Statistical analysis. The data are presented as the mean \pm SD and were analyzed using GraphPad Prism software, version 8.0 (GraphPad Software, Inc.). Statistical significance was determined using one-way ANOVA followed by Tukey's post hoc test for multiple groups, and unpaired Student's t-test for two groups. Each experiment was conducted $\geq 3$ times. $\mathrm{P}<0.05$ was considered to indicate a statistically significant difference.

\section{Results}

PDE4 expression in septic small intestinal tissue. The pathological changes in small intestinal tissue were observed following H\&E staining. The cells in the normal group were arranged in an orderly manner, and obvious intestinal villi and columnar cells could be observed at x200 magnification. However, the integrity of the intestinal tissue and the structure of villi were disrupted in the CLP group, and the arrangement of the cells became disordered. The number of vacuolar goblet cells increased significantly, accompanied by inflammatory cell infiltration (Fig. 1A). The levels of the intestinal indicators $\mathrm{LDH}, \mathrm{DAO}$ and iFABP were detected using corresponding kits and were found to be low in normal tissues and high in damaged intestinal tissues (Fig. 1B). RT-qPCR analysis (Fig. 1C) and western blotting (Fig. 1D) were employed to detect the expression level of PDE4. All three detected subtypes of PDE4 were elevated in the damaged tissue, although the difference in the expression of PDE4D was not as significant as that of the other two subtypes.

PDE4 expression in small intestinal cells. IEC-6 cells were treated with different concentrations of LPS for $24 \mathrm{~h}$ to detect its effect on cell viability. It was found that the cell survival rate decreased significantly with increasing LPS concentrations (Fig. 2A). Moreover, RT-qPCR analysis (Fig. 2B) and western blotting (Fig. 2C) were conducted to detect the expression level of PDE4 in cells treated with increasing doses of LPS. After treatment with different concentrations, it was observed that the expression level of PDE4 reached a peak at an LPS concentration of $10 \mu \mathrm{g} / \mathrm{ml}$. However, when the concentration was increased to $50 \mu \mathrm{g} / \mathrm{ml}$, PDE4 expression was decreased. This may be due to the fact that the majority of cells were necrotic. In the subsequent experiments, the cells were treated with LPS at a concentration of $10 \mu \mathrm{g} / \mathrm{ml}$, and the cell survival rate was maintained at $\sim 50 \%$.

Intestinal indicators reflect the effect of roflumilast on LPS-induced cells. RT-qPCR analysis (Fig. 3A) and western blotting (Fig. 3B) were conducted to detect the expression levels of PDE4A and PDE4B in cells treated with LPS or LPS + roflumilast. The expression levels of these factors were decreased in the LPS + roflumilast group compared with the LPS alone group. Furthermore, LDH, DAO and iFABP levels were detected using respective assay kits. In the LPS-induced group, the levels of these three factors were significantly increased. However, the addition of roflumilast suppressed their levels, and this inhibitory effect was more notable with a high concentration of roflumilast (Fig. 3C).

Roflumilast suppresses LPS-induced intestinal cell injury and release of inflammatory factors. The survival rate of the cells in the control group, the LPS induction group and the roflumilast groups was examined using a CCK-8 assay. The cell survival rate of the roflumilast groups was higher compared with that of the induction group (Fig. 4A). In addition, RT-qPCR analysis was used to detect the expression levels of the inflammation-related factors TNF- $\alpha$, IL- $1 \beta$, IL-6 and $\mathrm{C}-\mathrm{C}$ motif chemokine ligand 2 , and it was found that the expression levels of these inflammatory factors were increased in the LPS induction group, while they were inhibited after treatment with roflumilast (Fig. 4B). Western blotting was used to detect the expression level of NF- $\mathrm{NB}$ p65 in the nucleus and cytoplasm. It was observed that NF- $\kappa \mathrm{B}$ p65 was mainly expressed in the cytoplasm in normal cells, and was mainly localized to the nucleus after cells were exposed to LPS. Following treatment with roflumilast, the expression of $\mathrm{NF}-\kappa \mathrm{B}$ p65 in the nucleus was decreased and its expression in 
A
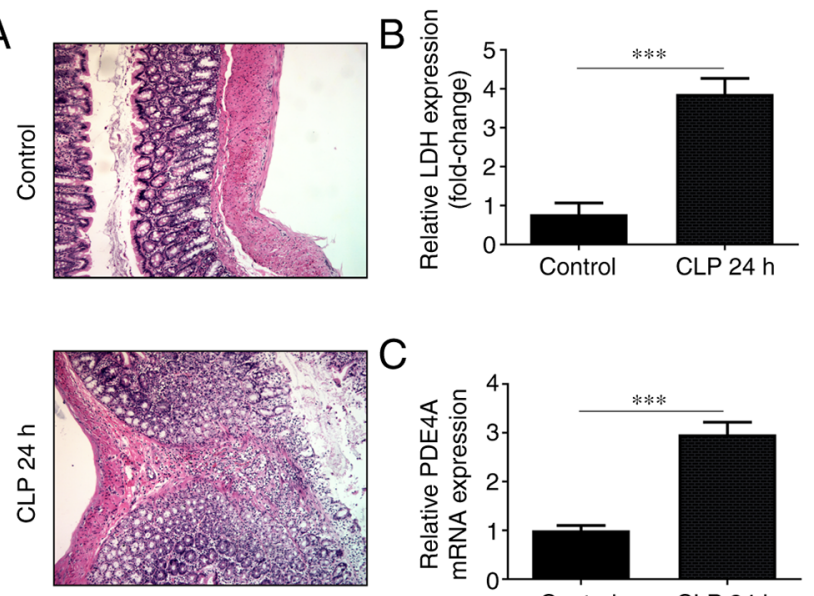

C
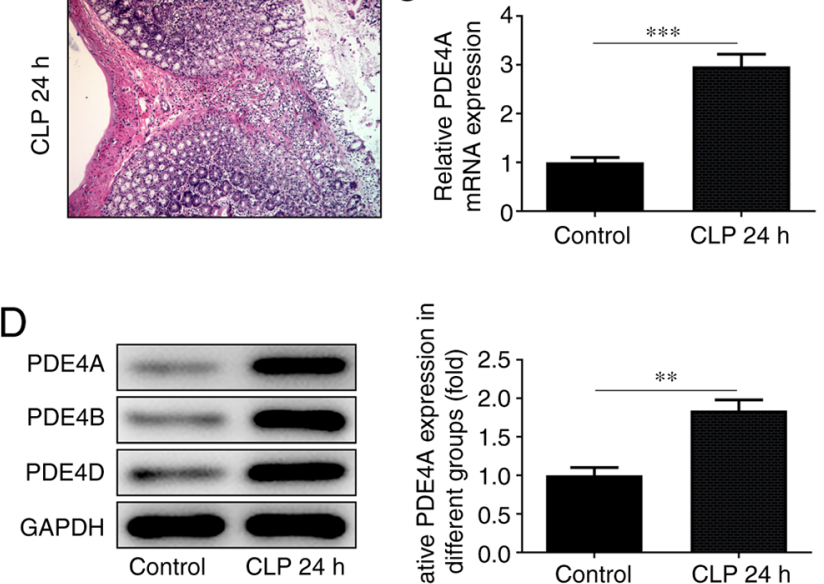
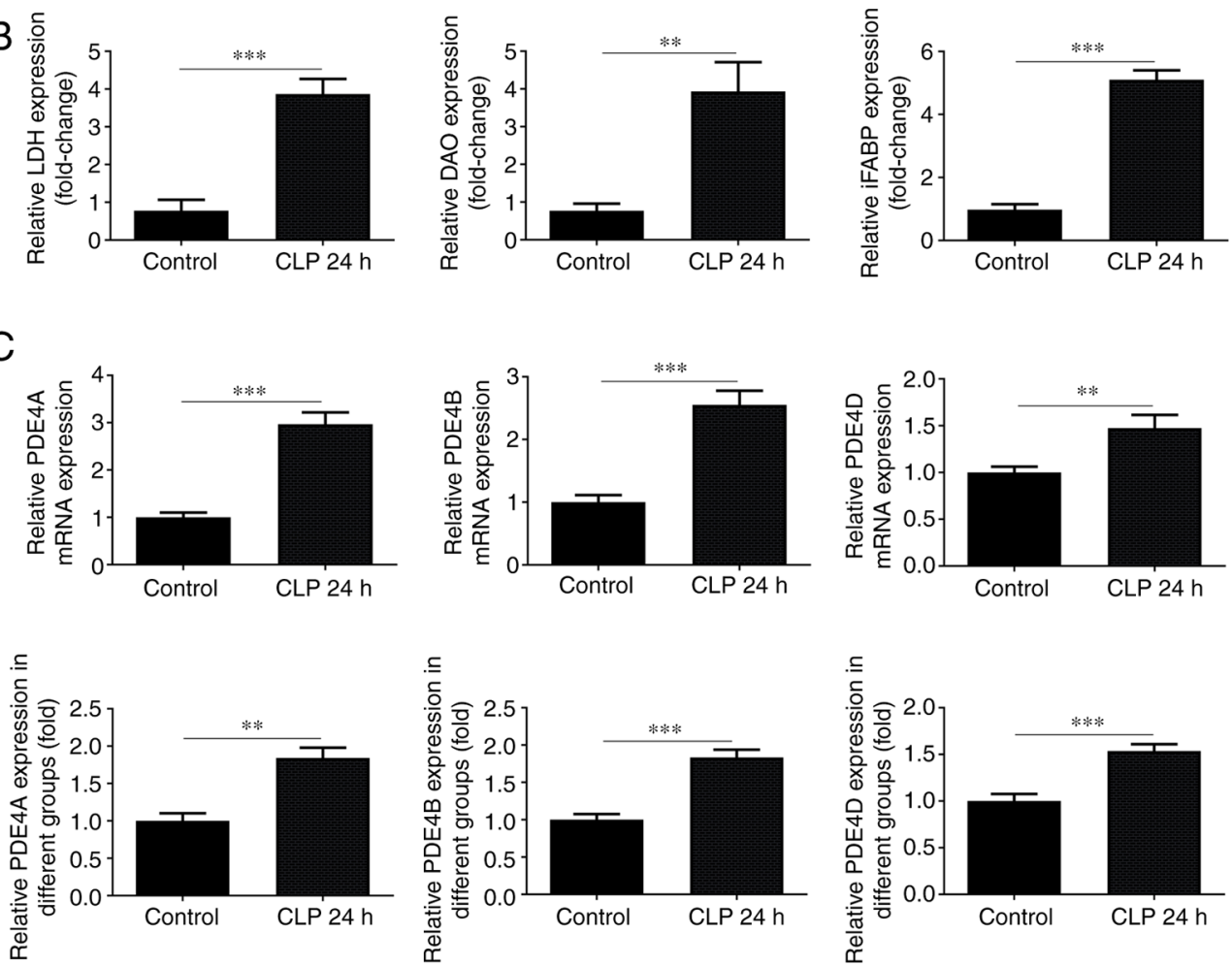

Figure 1. PDE4 expression in septic small intestinal tissue. (A) H\&E staining was used to observe the pathological changes in small intestinal tissue (magnification, x200). (B) LDH, DAO and iFABP levels in septic small intestinal tissue were detected using respective assay kits. (C) Reverse transcription-quantitative PCR analysis and (D) western blotting were conducted to detect the expression levels of PDE4A, PDE4B and PDE4D in septic small intestinal tissue. ${ }^{* *} \mathrm{P}<0.01,{ }^{* * *} \mathrm{P}<0.001 ; \mathrm{n} \geq 3$. PDE4, phosphodiesterase 4; LDH, lactate dehydrogenase; DAO, D-amino acid oxidase; iFABP, intestinal fatty acid binding protein; CLP, cecal ligation and puncture.

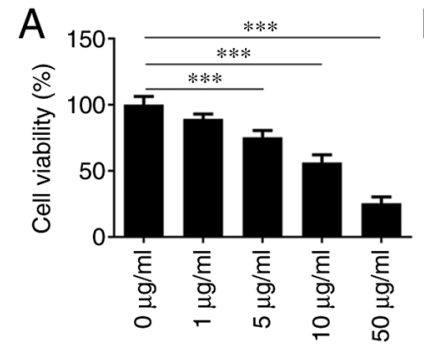

C



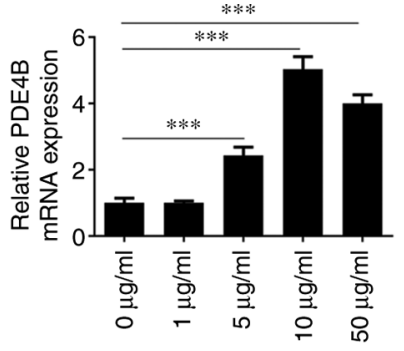

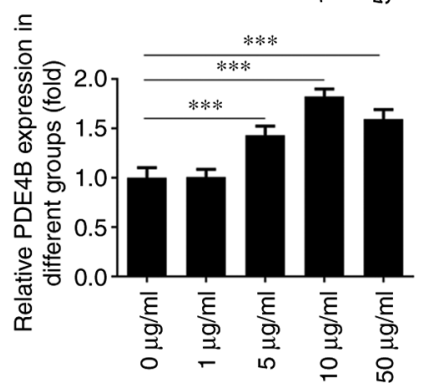

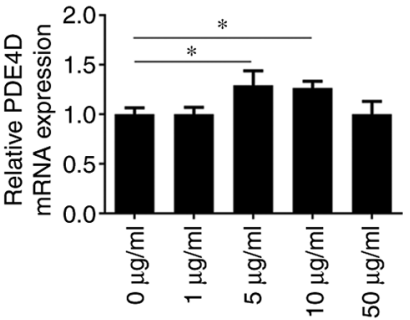

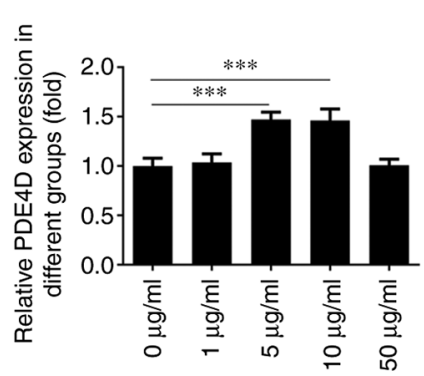

Figure 2. PDE4 expression in small intestinal cells. (A) IEC-6 cells were treated with different concentrations of LPS for $24 \mathrm{~h}$ to detect its effect on cell viability. (B) Reverse transcription-quantitative PCR analysis and (C) western blotting were performed to detect the expression levels of PDE4A, PDE4B and PDE4D in cells treated with increasing doses of LPS. "P $<0.05,{ }^{* * * *} \mathrm{P}<0.001 ; \mathrm{n} \geq 3$. PDE4, phosphodiesterase 4; LPS, lipopolysaccharide.

the cytoplasm was increased, which suggested that roflumilast relieved the activation of NF- $\mathrm{BB}$ p65 (Fig. 4C).

Roflumilast suppresses LPS-induced intestinal cell apoptosis. TUNEL staining was used to detect cell apoptosis. The fluorescence of the cells in the LPS group was significantly decreased compared with that of the control group, and the fluorescence of the roflumilast-treated groups was restored to varying degrees (Fig. 5A and B). Western blotting was performed to detect the expression levels of the apoptosis-related proteins Bax, cleaved caspase 3, cleaved PAPR and Bcl-2. The expression of the anti-apoptotic Bcl-2 protein was decreased in the LPS 

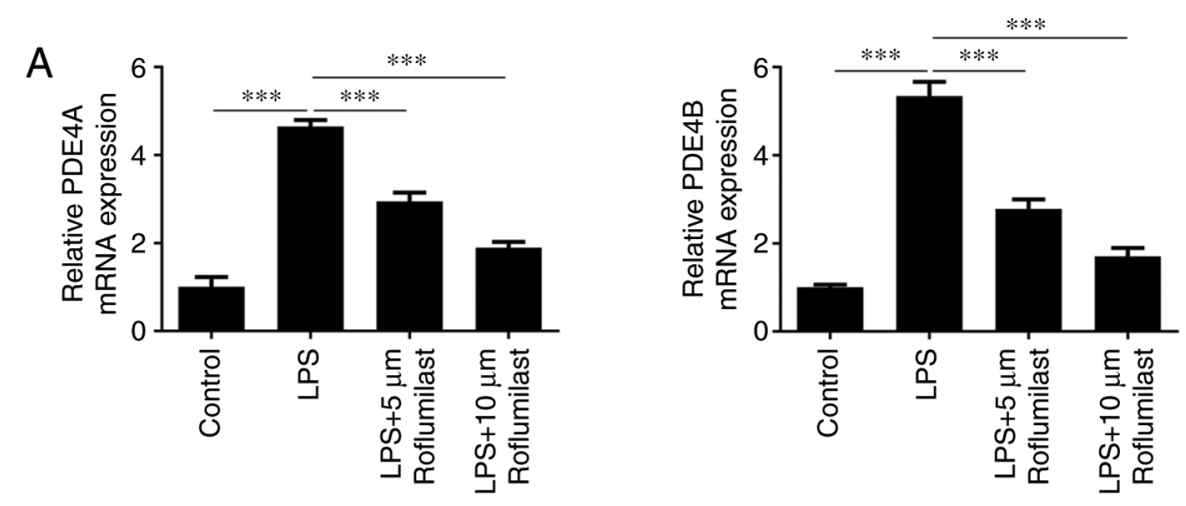

B
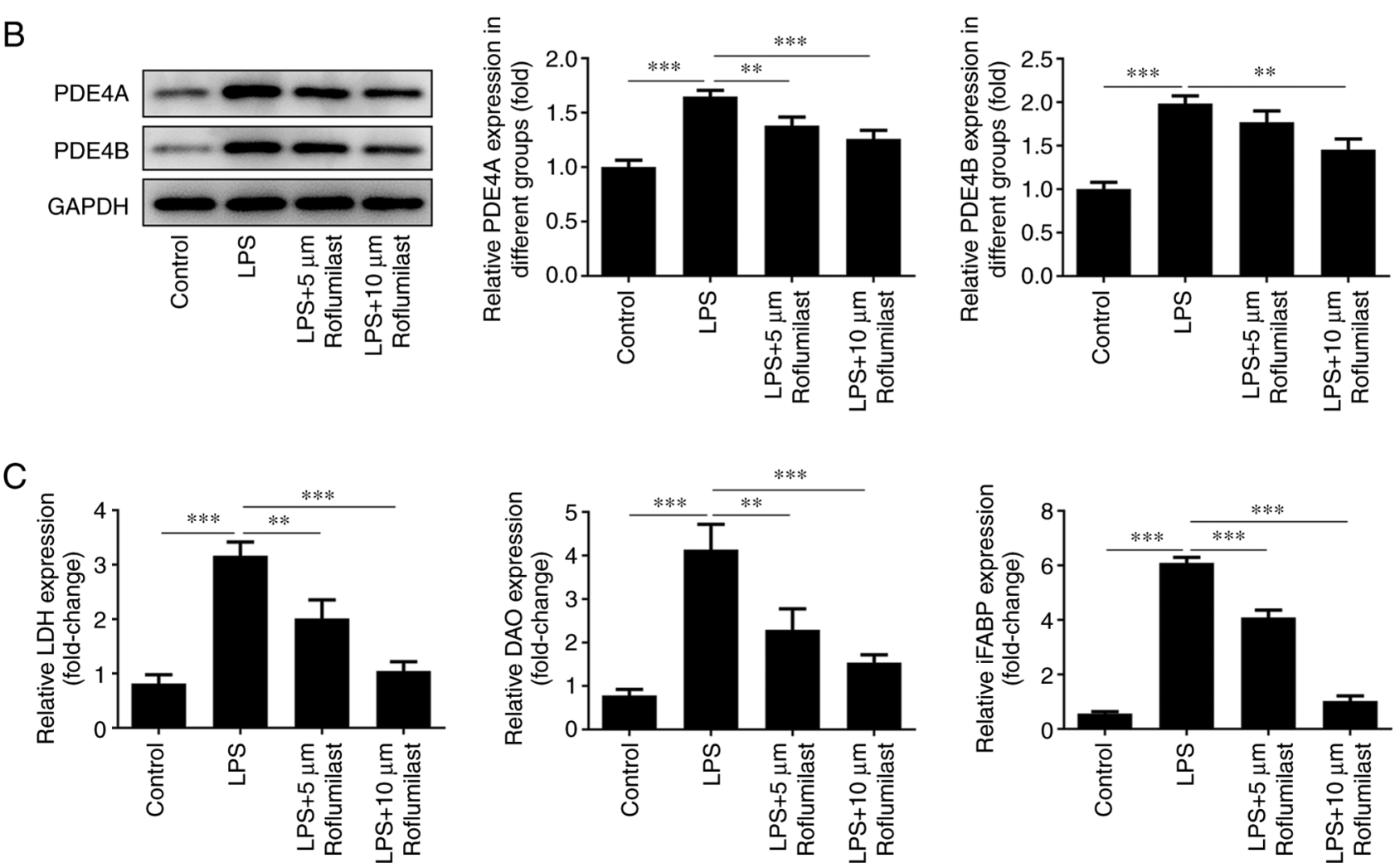

Figure 3. Intestinal indicators reflect the effect of roflumilast on LPS-induced cells. (A) Reverse transcription-quantitative PCR analysis and (B) western blotting were conducted to detect the expression levels of PDE4A and PDE4B in cells treated with LPS or LPS + roflumilast. (C) LDH, DAO and iFABP levels in cells were detected using respective assay kits. ${ }^{* *} \mathrm{P}<0.01,{ }^{* * *} \mathrm{P}<0.001 ; \mathrm{n} \geq 3$. PDE4, phosphodiesterase 4; LPS, lipopolysaccharide; LDH, lactate dehydrogenase; $\mathrm{DAO}, \mathrm{D}$-amino acid oxidase; iFABP, intestinal fatty acid binding protein.

induction group, but was increased in the roflumilast groups. The changing trend in the expression levels of other genes that promote apoptosis was the opposite, indicating that roflumilast reduces the apoptosis of LPS-induced cells (Fig. 5C).

\section{Discussion}

The mortality rate of sepsis remains the leading cause of disease-related mortality in intensive care medicine (19). Two main mechanisms underlying sepsis-induced gastrointestinal functional damage are currently known. The first mechanism is direct damage. For example, inflammation directly acts on the gastrointestinal mucosa, causing congestion and damage to the tight junctions between cells, resulting in intestinal mucosal damage (20). The second mechanism is indirect damage, involving insufficient blood supply to the intestines and malnutrition (21). There are numerous hypotheses regarding the mechanism of sepsis caused by gastrointestinal injury $(22,23)$, some of which have been confirmed (23); however, the exact mechanism remains to be determined. More importantly, treatment strategies targeting PDE4 have been attracting increased attention, and the expression levels of PDE4A, PDE4B and PDE4D have all been found to be increased in intestinal tissues (24). In view of the anti-inflammatory effects of the PDE inhibitor roflumilast, the current study was undertaken to examine whether roflumilast can relieve sepsis-induced intestinal damage.

In order to confirm the construction of the rat model of intestinal tissue injury and cell damage, certain indicators were evaluated. LDH is mainly found in cardiomyocytes, liver cells and skeletal muscle cells. Damage to these cells will lead to excessive LDH release, which is an indicator of tissue 
A
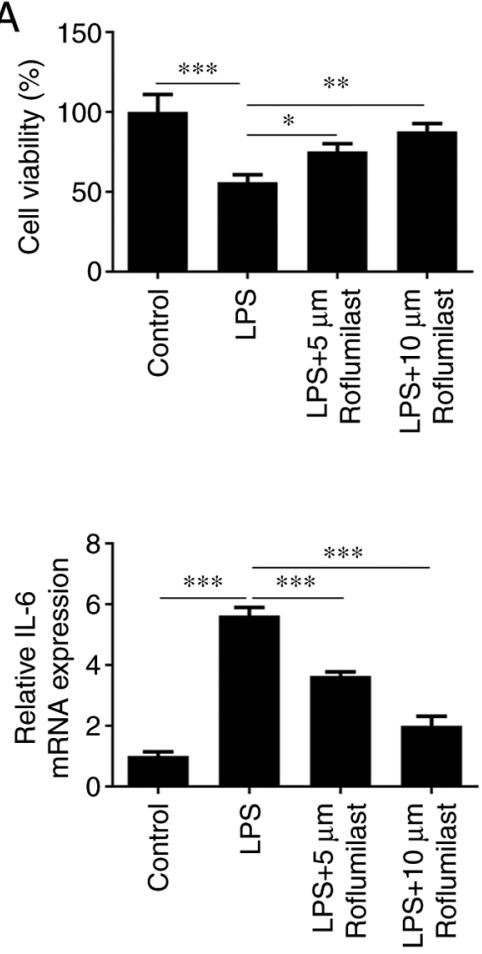

B
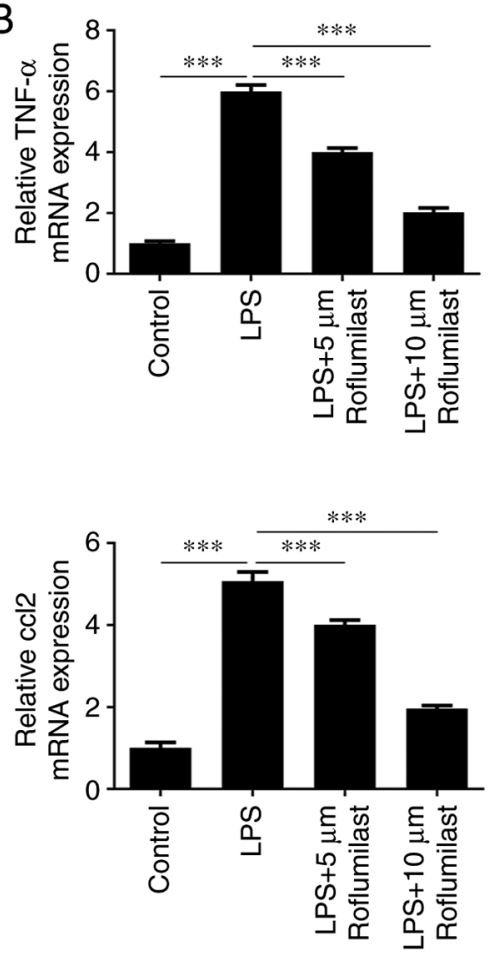

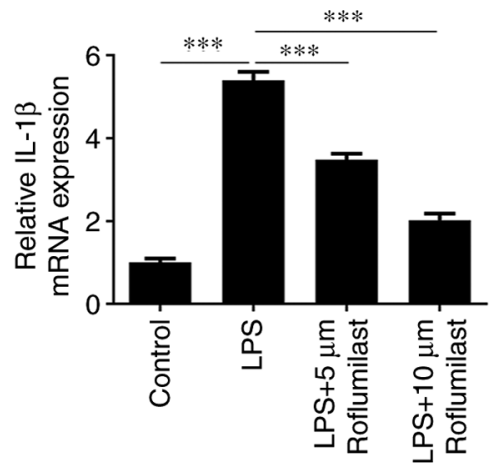

C

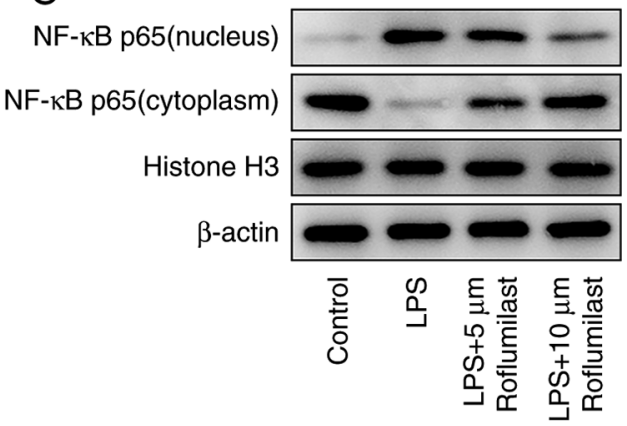

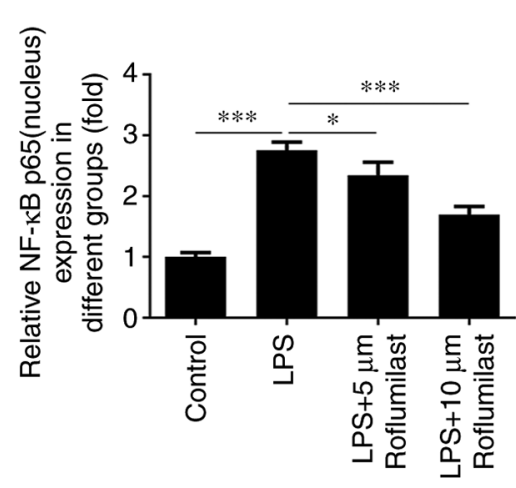
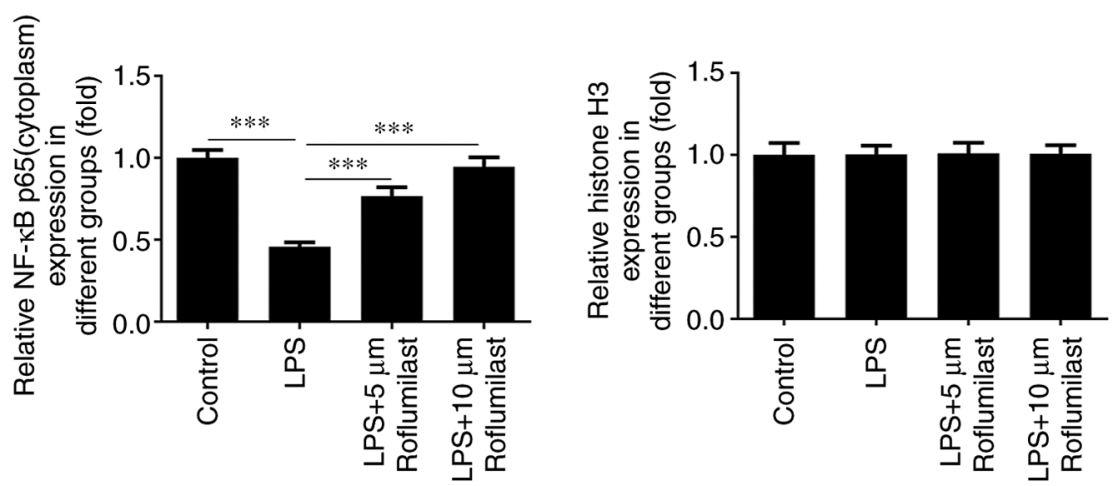

Figure 4. Roflumilast suppresses LPS-induced intestinal cell damage and release of inflammatory factors. (A) Survival rate of the cells in the control, LPS induction and roflumilast groups was examined using a Cell Counting Kit-8 assay. (B) Reverse transcription-quantitative PCR analysis was used to detect the expression levels of the inflammatory factors TNF- $\alpha$, IL-1 $\beta$, IL-6 and CCL2. (C) Western blotting was used to detect the expression level of NF- $k B$ p65 in the nucleus and cytoplasm. ${ }^{*} \mathrm{P}<0.05,{ }^{* *} \mathrm{P}<0.01,{ }^{* * *} \mathrm{P}<0.001 ; \mathrm{n} \geq 3$. CCL2, C-C motif chemokine ligand 2; LPS, lipopolysaccharide.

damage (25). The results of the present study demonstrated that the LDH levels were increased in the injury group, while roflumilast reduced LDH levels. DAO is an intracellular enzyme found in the small intestinal mucosa or ciliated epithelial cells, which can promote cell repair and protect the intestinal mucosa, and its levels increase when the mucosal barrier of the small intestine is damaged (26). Therefore, the activity of DAO may reflect the extent of intestinal damage. iFABP is specifically present in large quantities in the epithelial cells of the mucosal layer of small intestinal tissue. Following mucosal injury, iFABP is quickly released into the circulation, serving as an indicator of intestinal epithelial cell damage (27). The results of the present study revealed that roflumilast reduced the levels of these two indicators, suggesting that it may alleviate intestinal tissue damage and epithelial cell damage caused by sepsis. In addition, the expression level of PDE4 in septic intestinal tissue was upregulated, suggesting that there may be an association between PDE4 and sepsis-induced intestinal injury. Furthermore, the effect of dobutamine, which is used to treat septic shock, was reduced in septic mice suffering from CLP due to the upregulation of PDE4 (28), which appears to confirm this hypothesis.

Roflumilast has been used in the clinical treatment of COPD, and there are multiple preclinical studies being conducted (14-16). In the present study, roflumilast was found to alleviate the intestinal damage caused by sepsis. Moreover, medical researchers observed that the combination of roflumilast with cisplatin could inhibit the proliferation of ovarian cancer cells, induce their apoptosis and arrest the cell cycle in the $\mathrm{G}_{0} / \mathrm{G}_{1}$ phase (29). Takano et al (30) reported that PDE4 inhibition can improve the cognitive function of rhesus monkeys via the intravenous injection of roflumilast, while Peng et al (31) revealed that roflumilast can increase the viability of sevoflurane-treated rat neurons and reduce the 
A
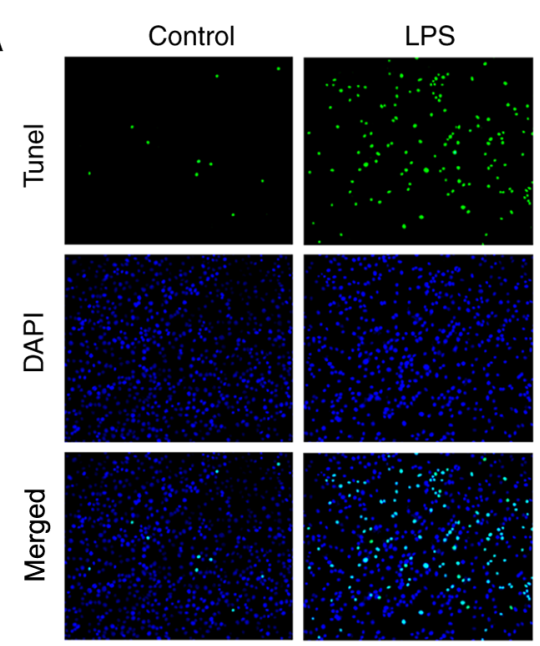

C

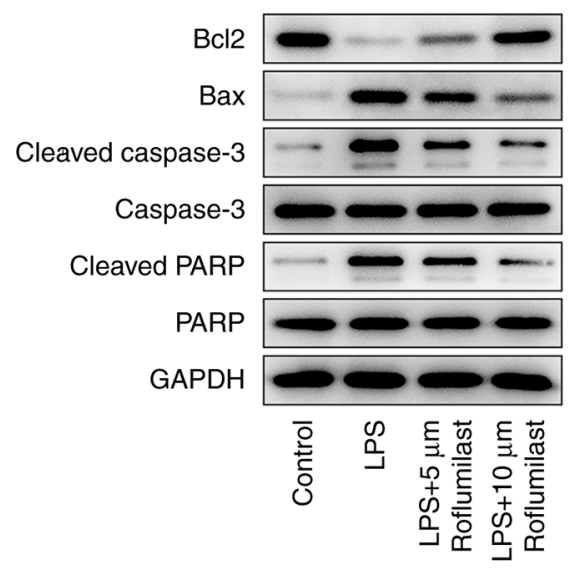

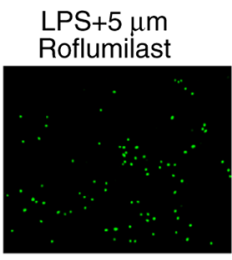
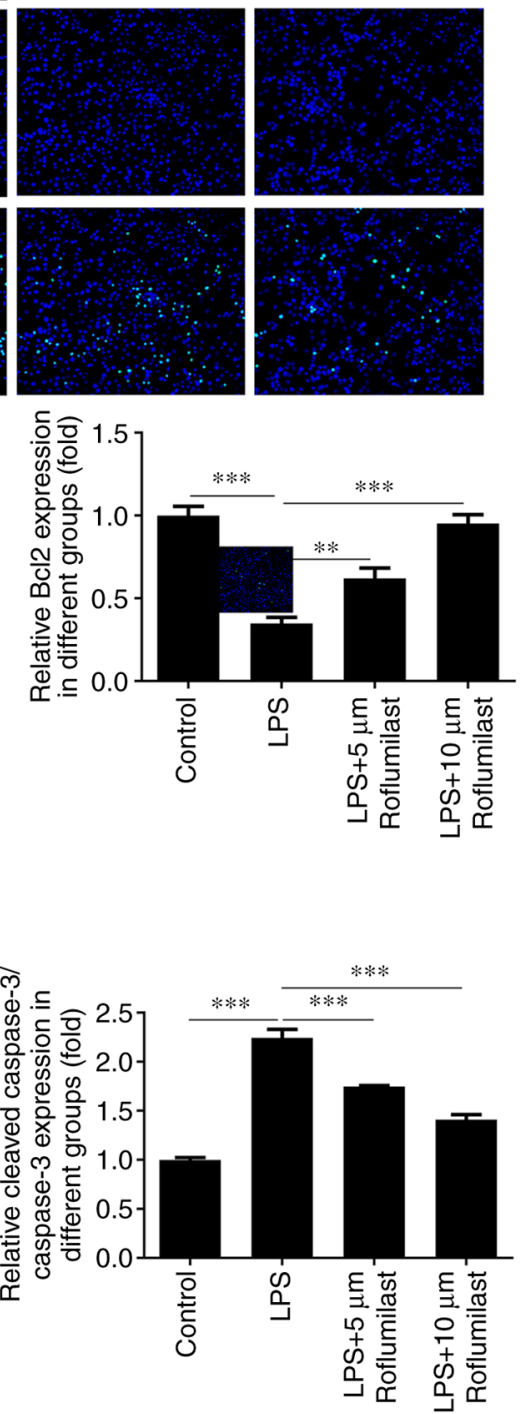

B


Figure 5. Roflumilast suppresses LPS-induced intestinal cell apoptosis. (A) TUNEL staining was used to detect cell apoptosis (magnification, x200). (B) Apoptosis rate in different groups is presented in the bar graph. (C) Western blotting was conducted to detect the expression levels of the apoptosis-related proteins Bax, cleaved caspase 3, cleaved PARP and Bcl2. ${ }^{* *} \mathrm{P}<0.01,{ }^{* * *} \mathrm{P}<0.001 ; \mathrm{n} \geq 3$. PARP, poly(ADP-ribose) polymerase 1; LPS, lipopolysaccharide.

level of apoptosis. In addition, El-Ashmawy et al (17) observed that roflumilast can increase the length of healthy colon in a rat model of ulcerative colitis, improve the histological score of the colon and thereby alleviate the severity of colitis. The present study provided a novel research direction for the clinical application of roflumilast based on the discovery of its effects in alleviating intestinal damage caused by sepsis.

In conclusion, cell injury is closely associated with inflammation, ultimately resulting in cell apoptosis. To the best of our knowledge, the present study was the first to demonstrate that roflumilast can reduce inflammatory factor release and cell apoptosis during intestinal damage resulting from sepsis. Moreover, roflumilast was found to downregulate PDE4 expression in LPS-induced intestinal cells, indicating that it may target PDE4 to reduce the intestinal damage caused by sepsis. The results of the present study uncovered the role of roflumilast in septic intestinal injury, and may help expand the scope of the clinical use of roflumilast; however, whether roflumilast inhibits intestinal injury by targeting PDE4 requires further in-depth investigation, and will be the focus of research in future studies. Furthermore, its application in the clinical treatment of intestinal injury requires numerous additional preclinical studies to verify its effects and elucidate the underlying mechanisms.

\section{Acknowledgements}

Not applicable. 


\section{Funding}

No funding was received.

\section{Availability of data and materials}

The datasets used and/or analyzed during the current study are available from the corresponding author on reasonable request.

\section{Authors' contributions}

$\mathrm{ZZ}$ conceived the study and wrote the manuscript. ML participated in the experiments and revised the manuscript. XW participated in the experiments and data processing. All authors have read and approved the final manuscript. ZZ and ML confirm the authenticity of the raw data.

\section{Ethics approval and consent to participate}

All procedures performed followed the ethical guidelines of Tianyou Hospital Affiliated to Wuhan University of Science \& Technology (Wuhan, China). All efforts were made to minimize animal suffering. The Institutional Animal Care and Use Committee of Tianyou Hospital Affiliated to Wuhan University of Science \& Technology has approved this study (June 2018-June 2021; approval no. 20180602).

\section{Patient consent for publication}

Not applicable.

\section{Competing interests}

The authors declare that they have no competing interests.

\section{References}

1. Faix JD: Biomarkers of sepsis. Crit Rev Clin Lab Sci 50: 23-36, 2013.

2. Huang M, Cai S and Su J: The pathogenesis of sepsis and potential therapeutic targets. Int J Mol Sci 20: 5376, 2019.

3. Martin GS: Sepsis, severe sepsis and septic shock: Changes in incidence, pathogens and outcomes. Expert Rev Anti Infect Ther 10: 701-706, 2012.

4. Rossaint $\mathrm{J}$ and Zarbock A: Pathogenesis of multiple organ failure in sepsis. Crit Rev Immunol 35: 277-291, 2015.

5. Huang CT, Tsai YJ, Tsai PR, Yu CJ and Ko WJ: Epidemiology and outcome of severe sepsis and septic shock in surgical intensive care units in Northern Taiwan. Medicine (Baltimore) 94: e2136, 2015.

6. Martinez A and Gil C: cAMP-specific phosphodiesterase inhibitors: Promising drugs for inflammatory and neurological diseases. Expert Opin Ther Pat 24: 1311-1321, 2014.

7. Lynch MJ,Hill EV and Houslay MD: Intracellular targeting of phosphodiesterase-4 underpins compartmentalized cAMP signaling. Curr Top Dev Biol 75: 225-259, 2006.

8. Ioakeimidis N and Kostis JB: Pharmacologic therapy for erectile dysfunction and its interaction with the cardiovascular system. J Cardiovasc Pharmacol Ther 19: 53-64, 2014.

9. Peng T, Qi B, He J, Ke H and Shi J: Advances in the development of phosphodiesterase-4 inhibitors. J Med Chem 63: 10594-10617, 2020.

10. Di Luigi L, Sansone M, Sansone A, Ceci R, Duranti G, Borrione P, Crescioli C, Sgrò P and Sabatini S: Phosphodiesterase type 5 inhibitors, sport and doping. Curr Sports Med Rep 16: 443-447, 2017.

11. Xu M, Yu X, Meng X, Huang S, Zhang Y, Zhang A and Jia Z: Inhibition of PDE4/PDE4B improves renal function and ameliorates inflammation in cisplatin-induced acute kidney injury. Am J Physiol Renal Physiol 318: F576-F588, 2020.
12. Sakkas LI, Mavropoulos A and Bogdanos DP: Phosphodiesterase 4 inhibitors in immune-mediated diseases: Mode of action, clinical applications, current and future perspectives. Curr Med Chem 24: 3054-3067, 2017.

13. Myers SA, Gobejishvili L, Saraswat Ohri S, Garrett Wilson C, Andres KR, Riegler AS, Donde H, Joshi-Barve S, Barve S and Whittemore SR: Following spinal cord injury, PDE4B drives an acute, local inflammatory response and a chronic, systemic response exacerbated by gut dysbiosis and endotoxemia. Neurobiol Dis 124: 353-363, 2019.

14. Feng H, Chen J, Wang H, Cheng Y, Zou Z, Zhong Q and Xu J: Roflumilast reverses polymicrobial sepsis-induced liver damage by inhibiting inflammation in mice. Lab Invest 97: 1008-1019, 2017.

15. Chang X, Hu LF, Ma XJ, Yin J, Liu XY and Li JB: Influence of roflumilast on sepsis mice through the JAK/STAT signaling pathway. Eur Rev Med Pharmacol Sci 23: 1335-1341, 2019.

16. Xu X, Liao L, Hu B, Jiang $\mathrm{H}$ and Tan M: Roflumilast, a phosphodiesterases-4 (PDE4) inhibitor, alleviates sepsis-induced acute kidney injury. Med Sci Monit 26: e921319, 2020.

17. El-Ashmawy NE, Khedr NF, El-Bahrawy HA and El-Adawy SA: Roflumilast, type 4 phosphodiesterase inhibitor, attenuates inflammation in rats with ulcerative colitis via down-regulation of iNOS and elevation of cAMP. Int Immunopharmacol 56: 36-42, 2018.

18. Livak KJ and Schmittgen TD: Analysis of relative gene expression data using real-time quantitative PCR and the 2(-Delta Delta C(T)) method. Methods 25: 402-408, 2001.

19. Stoller J, Halpin L, Weis M, Aplin B, Qu W, Georgescu C and Nazzal M: Epidemiology of severe sepsis: 2008-2012. J Crit Care 31: $58-62,2016$.

20. Sertaridou E, Papaioannou V, Kolios G and Pneumatikos I: Gut failure in critical care: Old school versus new school. Ann Gastroenterol 28: 309-322, 2015.

21. Yang H, Song Z, Jin H, Cui Y, Hou M and Gao Y: Protective effect of rhBNP on intestinal injury in the canine models of sepsis. Int Immunopharmacol 19: 262-266, 2014.

22. Deitch EA: Bacterial translocation or lymphatic drainage of toxic products from the gut: What is important in human beings? Surgery 131: 241-244, 2002.

23. Deitch EA, Xu D and Kaise VL: Role of the gut in the development of injury- and shock induced SIRS and MODS: The gut-lymph hypothesis, a review. Front Biosci 11: 520-528, 2006.

24. Li H, Fan C, Feng C, Wu Y, Lu H, He P, Yang X, Zhu F, Qi Q, Gao Y, et al: Inhibition of phosphodiesterase-4 attenuates murine ulcerative colitis through interference with mucosal immunity. Br J Pharmacol 176: 2209-2226, 2019.

25. Kumar P, Nagarajan A and Uchil PD: Analysis of cell viability by the lactate dehydrogenase assay. Cold Spring Harb Protoc 2018, 2018.

26. Wolvekamp MC and de Bruin RW: Diamine oxidase: An overview of historical, biochemical and functional aspects. Dig Dis 12: 2-14, 1994.

27. Derikx JP, Schellekens DH and Acosta S: Serological markers for human intestinal ischemia: A systematic review. Best Pract Res Clin Gastroenterol 31: 69-74, 2017.

28. Sakai M, Suzuki T, Tomita K, Yamashita S, Palikhe S, Hattori K, Yoshimura N, Matsuda N and Hattori Y: Diminished responsiveness to dobutamine as an inotrope in mice with cecal ligation and puncture-induced sepsis: Attribution to phosphodiesterase 4 upregulation. Am J Physiol Heart Circ Physiol 312: H1224-H1237, 2017.

29. Gong S, Chen Y, Meng F, Zhang Y, Li C, Zhang G, Huan W and Wu F: Roflumilast enhances cisplatin-sensitivity and reverses cisplatin-resistance of ovarian cancer cells via cAMP/PKA/CREB-FtMt signalling axis. Cell Prolif 51: e12474, 2018.

30. Takano A, Uz T, Garcia-Segovia J, Tsai M, Lahu G, Amini N, Nakao R, Jia Z and Halldin C: A nonhuman primate PET study: Measurement of brain PDE4 occupancy by roflumilast using (R)- $\left[{ }^{11} \mathrm{C}\right]$ Rolipram. Mol Imaging Biol 20: 615-622, 2018.

31. Peng S, Yan HZ, Liu PR, Shi XW, Liu CL, Liu Q and Zhang Y: Phosphodiesterase 4 inhibitor roflumilast protects rat hippocampal neurons from sevoflurane induced injury via modulation of MEK/ERK signaling pathway. Cell Physiol Biochem 45: 2329-2337, 2018. International (CC BY-NC-ND 4.0) License. 